\title{
Three dimensional (3D) microstructure-based modeling of interfacial decohesion in particle reinforced metal matrix composites
}

\author{
J.J. Williams ${ }^{\text {a }}$, J. Segurado ${ }^{\mathrm{b}, \mathrm{c}}$, J. LLorca ${ }^{\mathrm{b}, \mathrm{c}}$, N. Chawla ${ }^{\mathrm{a}, *}$
}

\begin{abstract}
A B S T R A C T
Modeling and prediction of the overall elastic-plastic response and local damage mechanisms in heterogeneous materials, in particular particle reinforced composites, is a very complex problem. Microstructural complexities such as the inhomogeneous spatial distribution of particles, irregular morphology of the particles, and anisotropy in particle orientation after secondary processing, such as extrusion, significantly affect deformation behavior. We have studied the effect of particle/matrix interface debonding in $\mathrm{SiC}$ particle reinforced Al alloy matrix composites with (a) actual microstructure consisting of angular SiC particles and (b) idealized ellipsoidal SiC particles. Tensile deformation in SiC particle reinforced $\mathrm{Al}$ matrix composites was modeled using actual microstructures reconstructed from serial sectioning approach. Interfacial debonding was modeled using user-defined cohesive zone elements.

Modeling with the actual microstructure (versus idealized ellipsoids) has a significant influence on: (a) localized stresses and strains in particle and matrix, and (b) far-field strain at which localized debonding takes place. The angular particles exhibited higher degree of load transfer and are more sensitive to interfacial debonding. Larger decreases in stress are observed in the angular particles, because of the flat surfaces, normal to the loading axis, which bear load. Furthermore, simplification of particle morphology may lead to erroneous results.
\end{abstract}

\section{Introduction}

The design and development of high performance materials requires a thorough understanding and careful control of microstructure and its effect on properties. This is particularly challenging given the multiphase and heterogeneous nature of most high performance composites. Modeling and prediction of the overall elastic-plastic response and local damage mechanisms in heterogeneous materials, in particular particle reinforced composites, is a very complex problem.

In multiphase materials, numerical modeling techniques, such as finite element method (FEM), are often more effective than analytical modeling since these materials lack the structural simplicity of continuous fiber composites or laminates and hence are not readily amenable to closed-form theoretical analyses. Another advantage of numerical modeling is that deformation and damage characteristics, particularly on a local scale, can be revealed. Numerical modeling of the behavior of multiphase materials has typically been conducted by assuming a single fiber, whisker, or particle of simple geometry in a unit cell model [1-3]. Unit cell models have been employed to model fracture of the ceramic reinforcement [4-7], void nucleation, growth and coalescence of voids within the metallic matrix [8], and/or decohesion and crack growth along the particle/matrix interface [9].

Another important aspect of the microstructure in the composite is the effect of spatial distribution of the particles. The link between spatial distribution and mechanical behavior has not been modeled extensively. Ghosh and co-workers [10,11] used a serial sectioning technique to obtain the spatial distribution of the $\mathrm{SiC}$ particles, and quantified the spatial distribution by a tessellation scheme. Modeling of damage in the composite was conducted on 2D sections by approximating the particle morphology as ellipsoids, so the deformation assumed a two-dimensional stress state (plane stress, plane strain, or modified plane strain). A 3D elastic Voronoi cell is also being developed [12], once again using ellipsoid particles. Boselli et al. [13] modeled the effect of crack growth using idealized 2D microstructures, consisting of circular disks embedded in a metal matrix. It was found that clustering had a significant effect on the local shielding and "antishielding" effects at the crack tip. Llorca and co-workers [14] recently modeled the effect of particle clustering on damage in 
MMCs in 3D. The particles, modeled as spheres, were incorporated with different degrees of clustering (as quantified by a radial distribution function). Moreover, they included the effect of damage by interface decohesion or matrix failure by void growth and coalescence into the simulations [15-17]. It was found that while the average stress in the particles did not vary significantly with clustering, the standard deviation in stress did. Thus, it was shown that for a given far-field applied stress in highly clustered composites, a given particle locally may have a much higher stress deviation from the average stress, and, thus, be more prone to fracture. In addition, tensile hydrostatic stresses were higher in the highly clustered regions of the microstructure, leading to early nucleation of damage by interface decohesion and accelerated void growth in the matrix.

The survey of the modeling approaches and results in the literature indicate that while conventional numerical modeling is reasonable for modeling simple shapes, such as cylindrical fibers, the unit cell models approximate the highly variable and irregular angular structure of particles by using simplified particle geometries such as spheres, ellipsoids, or cubes. Thus, while simplifications in unit cell models may aid in computation, they fail to capture the complex morphology, size, and spatial distribution of the reinforcement. It follows that an accurate simulation of the material behavior can really only be obtained by incorporating actual 3D microstructures as a basis for the model. Chawla and co-workers [18-20] have developed and employed microstructure-based finite element techniques that are able to incorporate the "true" composite microstructures. These models consider the inherent particle morphology and clustering of particles, as a basis for analysis using finite element techniques, with minimal approximations.

Our initial work in this area concentrated on simple linear elastic analysis of two-dimensional (2D) microstructures [18]. Here, microstructural images from optical and/or scanning electron microscopy were segmented, and transformed into a vectorial format, for finite element analysis. More recently, the microstructure-based approach has included elastic-plastic analysis, whereby the microstructure is exported to a commercial finite element analysis software. Currently, models have been used to conduct 3D microstructure simulations, by obtaining $2 \mathrm{D}$ images from serial sectioning or $\mathrm{X}$-ray tomography, reconstruction of the 3D virtual microstructure, and incorporation of the 3D model into a FEM analysis [21]. In this manner, prediction of the macroscopic stress-strain behavior, as well as insight into the localized nature of deformation, can be obtained. It should be noted that other techniques, such as X-ray tomography [22-25] and holotomography [26], simulated microstructures [27-29], and multiphase elements [30] have also been incorporated into FEM analysis. Nevertheless, microstructure-based FEM have not incorporated the actual failure mechanisms observed in these materials (such as interface decohesion).
Microstructural complexities such as the inhomogeneous spatial distribution of particles, irregular morphology of the particles, and anisotropy in particle orientation after secondary processing, such as extrusion, significantly affect deformation behavior. Accurate prediction of macroscopic deformation behavior and an understanding of localized damage mechanisms can be accomplished by capturing the microstructure of the material as a basis for the model [31]. In this paper we examined the role of particle/matrix interfacial decohesion using actual microstructures, obtained from a 3D serial sectioning approach. Simplified models, consisting of perfect ellipsoids and spheres (while maintaining the distribution of the particles constant) were also employed. It will be shown that 3D microstructure-based modeling approaches provide a quantitative understanding of localized damage phenomena (which are not predicted in simplified models), as well as excellent corroboration of experimental observations.

\section{Materials and experimental procedure}

A 2080 aluminum alloy $(3.6 \% \mathrm{Cu}, 1.9 \% \mathrm{Mg}$, and $0.25 \% \mathrm{Zr}$ ) reinforced with 10,20 , and $30 \mathrm{vol} \% \mathrm{SiC}$ particles (with an average particle size of $8 \mu \mathrm{m}$ ) was used in this study. The materials were processed by the powder metallurgy technique (Alcoa Inc, Alcoa, PA). The matrix powder and reinforcement particles were blended, and the powder mixture canned and degassed to remove adsorbed water and/or other volatile elements. The material was then vacuum hot pressed and hot extruded [32]. The composites were electro-discharge machined (EDM) into rectangular blanks, solution treated at $493{ }^{\circ} \mathrm{C}$ for $2 \mathrm{~h}$, water quenched, and peak-aged at $175^{\circ} \mathrm{C}$ for $24 \mathrm{~h}$ (T6 condition).

Specimens were machined parallel to the extrusion axis. The following steps were used for the serial sectioning process and 3D visualization and modeling. The composite samples were cut and mounted. The first step was to choose a representative region of the microstructure. Selection of this region of interest is very important, but somewhat subjective. It is desirable to obtain a number of sections that encompass several $\mathrm{SiC}$ particles in a given volume, to allow entire particles to be reconstructed and incorporated in modeling. This volume is also dependent on the feature size (e.g., particle size). A volume of $100 \times 100 \times 20 \mu \mathrm{m}^{3}$ yields approximately 100 particles, assuming that the $\mathrm{SiC}$ particles are approximately $\sim 6-$ $8 \mu \mathrm{m}$ in diameter, with a volume fraction of $20 \% \mathrm{SiC}$. This volume served as a starting point for determining a "representative" number of particles for the FEM simulated response. The effect of microstructure volume on predicted deformation response of the composites was confirmed, to ensure that the volume being modeled was representative of the deformation behavior of the material. This was done by incrementally increasing the model size until the predicted response did not change. a

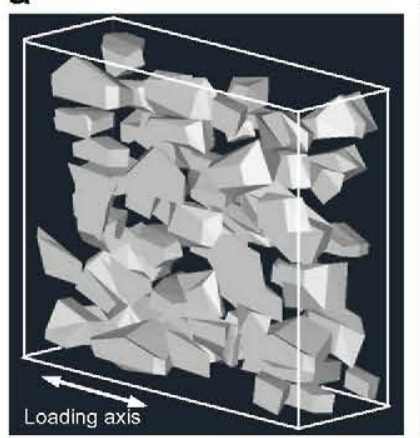

b

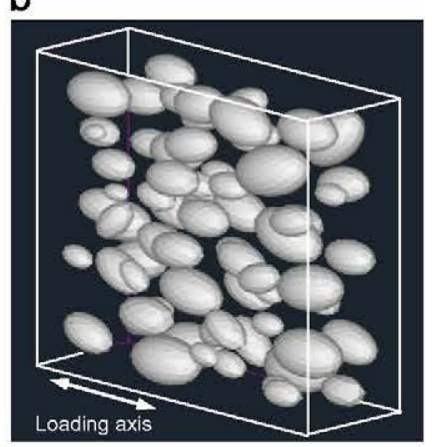

C

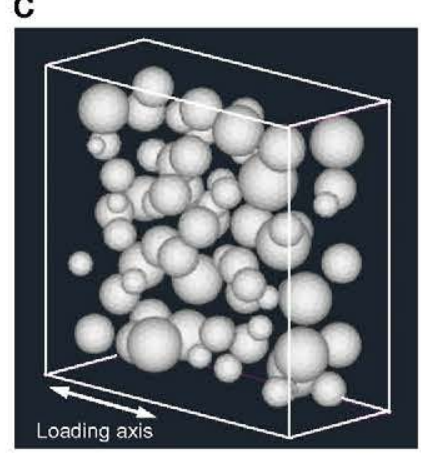

Fig. 1. 3D models of SiC particle reinforced $\mathrm{Al}$ (a) actual microstructure, (b) ellipsoid particles, and (c) spherical particles. 
Fiducial marks, made by Vickers indentation, were used to measure the material thickness loss during polishing/grinding. Since the geometry of the indenter is known, the amount of material thickness removed can be calculated. The average cumulative thickness loss rate was taken as the average of indentations at the four corners of the square region of interest. This rate was calibrated to give a thickness loss of about $1 \mu \mathrm{m} /$ cycle. Since the size of the microstructural features dictates the thickness between sections, with $\mathrm{SiC}$ particles about $8 \mu \mathrm{m}$ in diameter, this would yield at least 5-6 sections per particle.

Cyclic polishing and imaging of the sample surface were then conducted to generate a series of microstructural sections. The role of polishing in serial sectioning is very important, for two reasons: (a) to control the amount of material removed, and (b) to obtain a high quality surface finish for microstructural characterization. In order to obtain the desired $1 \mu \mathrm{m} /$ cycle target, the composite was polished with $1 \mu \mathrm{m}$ diamond paste for $20 \mathrm{~min}$ while applying a load of $5 \mathrm{~N}$. After each polishing cycle, images of the microstructure were taken with an optical microscope. The sample was secured using a mounting fixture to minimize translational and rotational misalignments between sections. The microstructures were segmented into black and white images using a conventional image analysis software (Image], Bethesda, MD).

The segmented microstructures were stacked and the 3D morphology of the particles was reconstructed using a vectoral format software (SurfDriver, University of Alberta \& University of Hawaii). The 3D particles were simplified slightly during reconstruction to aid in computation, although the simplifications did not significantly change the original morphology of the SiC particles.

\section{Results and discussion}

The finite element method (FEM) has been used to simulate the mechanical behavior of the materials under tensile loading. Fig. 1 shows two 3D models of the microstructure of extruded SiC particle reinforced Al alloy matrix composite. Fig. 1(a) shows the actual geometry of the SiC particles, while Fig. 1(b) shows the particles approximated as ellipsoidal particles. The tensile

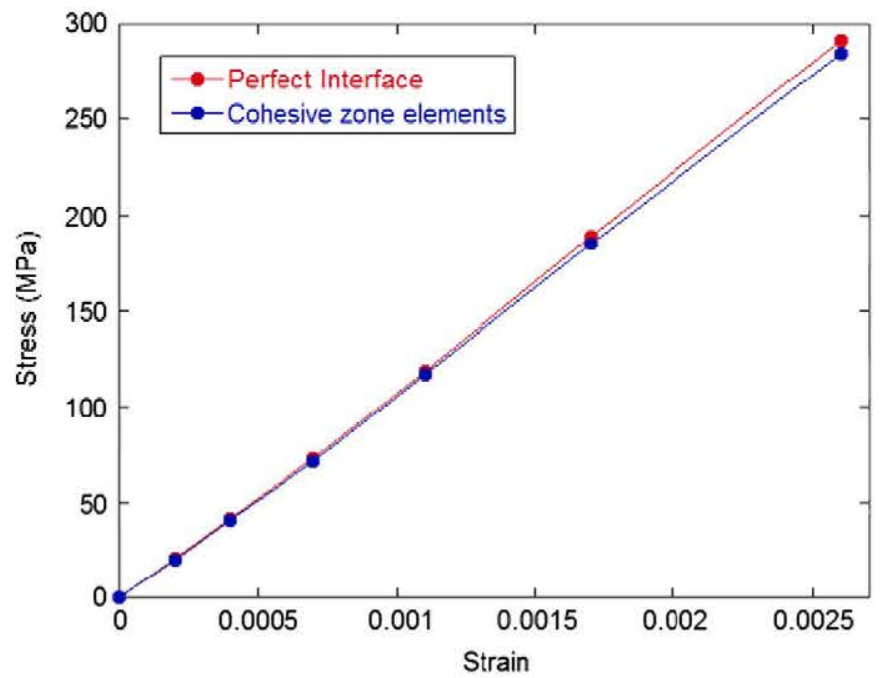

Fig. 4. Comparison of stress-strain response in the elastic region for a model with cohesive zone elements and a perfect interface. Prior to debonding the model response is the same.
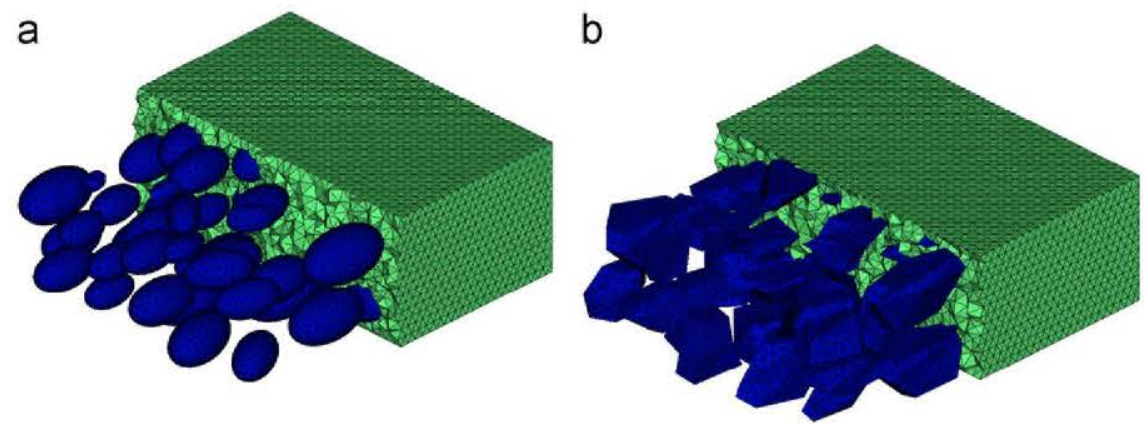

Fig. 2. Meshed 3D models of SiC particle reinforced $\mathrm{Al}$ (a) ellipsoid particles and (b) actual microstructure.
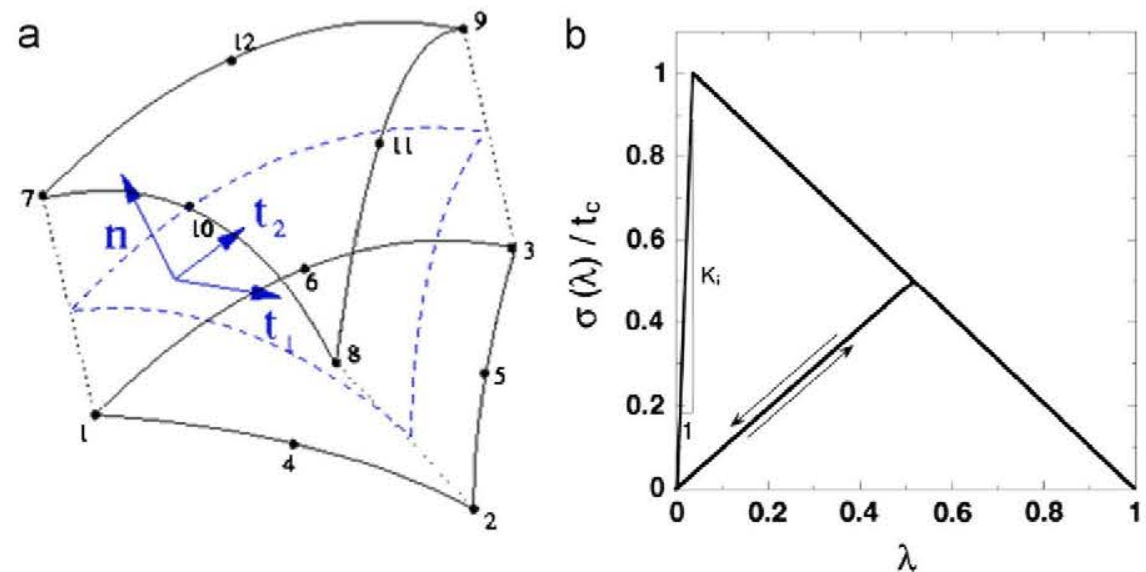

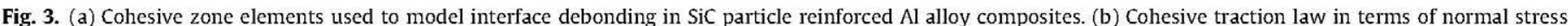
versus crack opening displacement. 
behavior of the two models was simulated using FEM. The refined 3D virtual model was exported into a pre-processor finite element meshing program (Hypermesh, Altair Engineering, Troy, MI) and then into an implicit commercial finite element analysis program (ABAQUS standard, Pawtucket, RI).

Rectangular prisms of dimensions $L 1 \times L 2 \times L 3$ were used as representative volume element, size that embeds approximately 100 particles. The model volume (matrix and reinforcing particles) was meshed using modified 10-node tetrahedral (C3D10M in Abaqus) using an adaptive automatic meshing algorithm. A cut of the final mesh together with the definition of the reference system is plotted in Fig. 2, being the direction 1 the loading axis. The modified elements contain an extra internal degree of freedom, and provide higher accuracy to reproduce the strain gradient in the

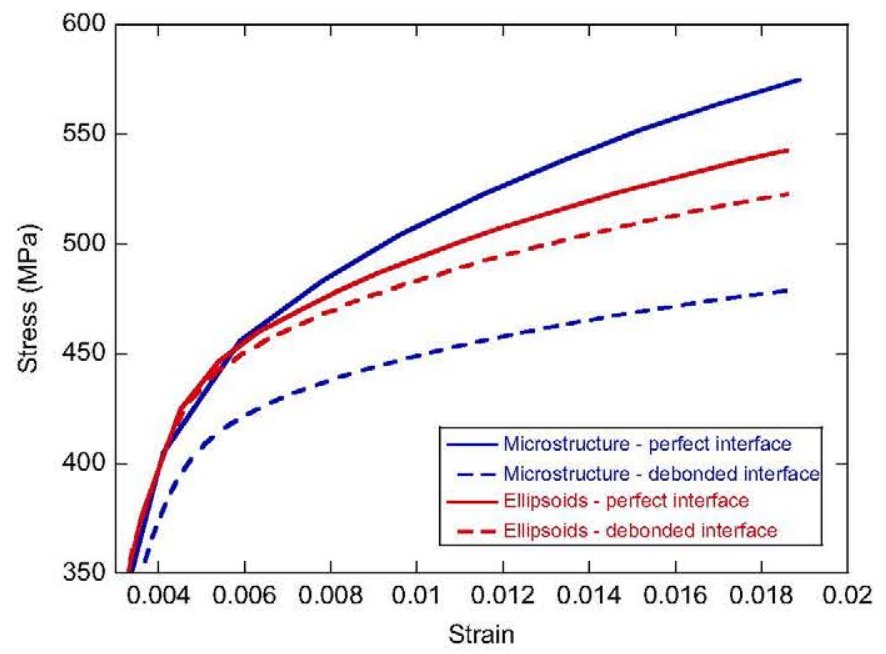

Fig. 5. Comparison of stress-strain response for (a) ellipsoidal particles and (b) angular particles. The effect of debonding is much more pronounced in the actual microstructure. matrix between closely-packed particles. Moreover, special care was taken to ensure that the matrix discretization between particles contained at least two element layers. A typical model comprised approximately 100,000-150,000 elements.

The particle behavior was assumed to be elastic with elastic modulus $E_{s}=410 \mathrm{GPa}$ and Poisson's ratio $v_{s}=0.20$. The matrix was modeled as an isotropically hardening elastic-plastic solid following the incremental (J2) theory of plasticity. The matrix elastic constants were $E_{m}=74 \mathrm{GPa}$ and $v_{m}=0.30$, and the isotropic matrix hardening during plastic deformation was given by the expression:

$\sigma_{m}^{e q}=A\left[\varepsilon_{m}^{p}\right]^{n}$

where $\sigma_{m}^{e q}$ is the Von Mises equivalent stress and $\varepsilon_{m}^{p}$ stands for the accumulated plastic strain. The constants $A=400 \mathrm{MPa}$ and $n=0.15$ are typical of an $\mathrm{Al}$ alloy.

In order to account for the damage due to particle decohesion, a cohesive crack model was introduced in the interfaces between particles and matrix $[15,17]$. The cohesive model enters into the simulation by interface elements of zero thickness connecting matrix and particle faces. Although cohesive interface elements are included in ABAQUS since version 6.5, none of the elements present in the program library is fully compatible with the elements of particles and matrix (C3D10M) and the user interface element developed by Segurado and LLorca [33] has been used for this purpose. The interface element consists of a double 6-noded triangular element with initial zero thickness (geometry depicted on Fig. 3(a)) where the normal and tangential stresses transferred by the interface were derived from a potential $\Phi$ given by:

$\Phi\left(\Delta u_{n}, \Delta u_{t 1}, \Delta u_{t 2}\right)=\Delta u_{c} \int_{0}^{\lambda} \sigma\left(\lambda^{\prime}\right) d \lambda^{\prime}$

where $\Delta u_{n}, \Delta u_{t 1}$ and $\Delta u_{t 2}$ stand for the normal and tangential relative displacements between the crack faces, and $\Delta u_{c}$ is the critical normal (or tangential) displacement between the crack faces at which all interactions vanish. $\lambda$ is the generalized crack
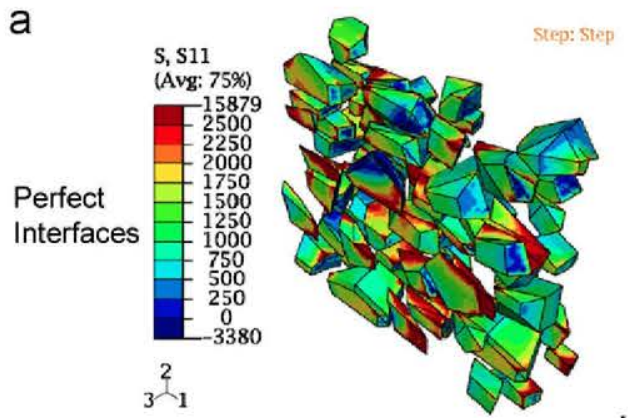

b
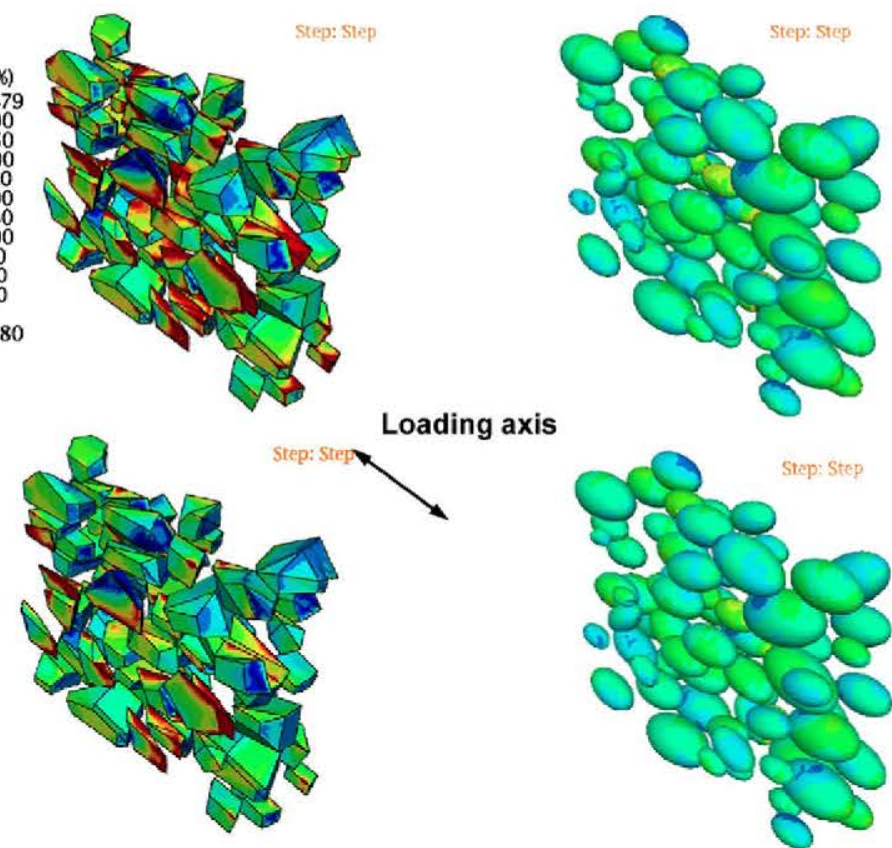

Debonding Interfaces

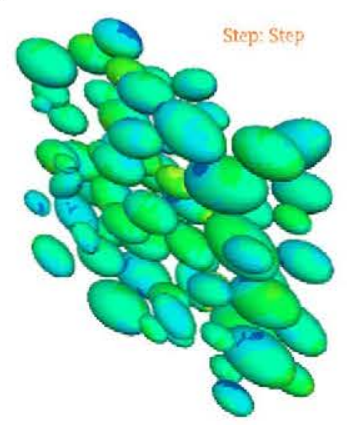

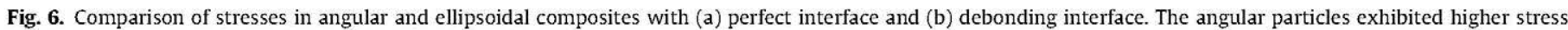
due to more load transfer, and relaxation after debonding. The macroscopic applied strain is $1.9 \%$. 
opening displacement expressed as

$\lambda=\sqrt{\left(\frac{\Delta u_{n}}{\Delta u_{c}}\right)^{2}+\left(\frac{\Delta u_{t 1}}{\Delta u_{c}}\right)^{2}+\left(\frac{\Delta u_{t 2}}{\Delta u_{c}}\right)^{2}}$

The particular function $\sigma(\lambda)$ (which stands for the normal stress transferred through the crack in the absence of tangential displacements) chosen for this simulation consists on a linear softening curve and is plotted in Fig. 3(b). The interface behavior is totally defined then by the two physical independent parameters: the interface strength, $t_{c}$, and the fracture energy, $\Gamma_{i}$, which is the area enclosed under the $\sigma(\lambda)$ function:

$\Gamma_{i}=\frac{1}{2} t_{c} \Delta u_{c}$
In addition, a numerical parameter defining the initial stiffness $K_{i}$ has to be introduced and adjusted to ensure that the presence of the interface elements does not perturb the stress fields around the spherical particles in the absence of damage.

The initial stiffness value has been set to $5 \times 10^{5} \mathrm{~N} / \mathrm{m}^{2}$, which in the absence of damage provides a macroscopic response almost identical to the same model without cohesive interfaces (Fig. 4) and does not affect the convergence of the problem too much . The critical displacement, $\Delta u_{c}$, used for all simulations was $1 \mu \mathrm{m}$ and the interface strength was set to different values $300 \mathrm{MPa}$, $500 \mathrm{MPa}, 3000 \mathrm{MPa}$, and a perfect interface (infinite strength). The boundary conditions applied to the RVEs in order to simulate uniaxial tension are defined in the reference system of Fig. 6 . The load is applied by imposing a displacement $\varepsilon_{1} L_{1}$, to each node on the face $x_{1}=L_{1}$, being $\varepsilon_{1}$ the value of the applied strain. All nodes

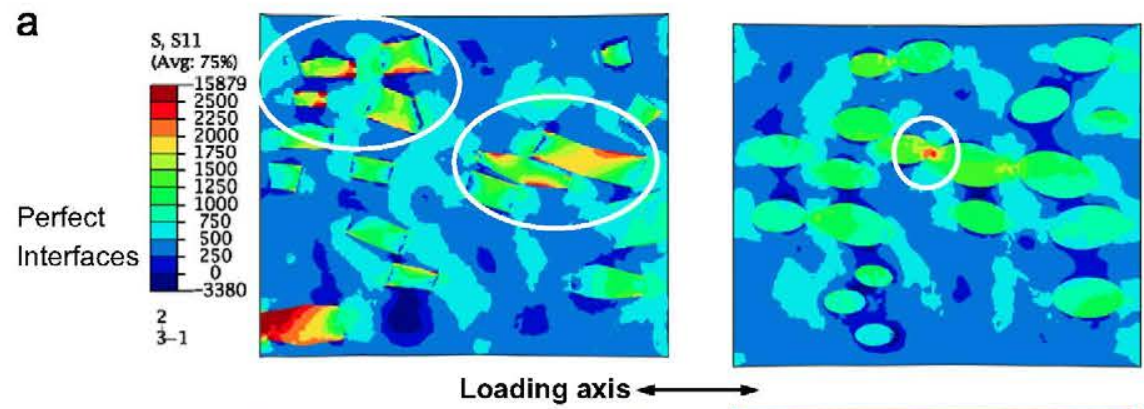

b

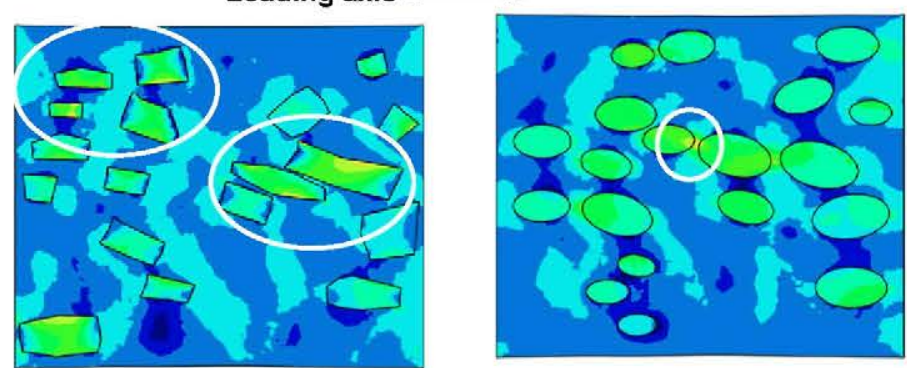

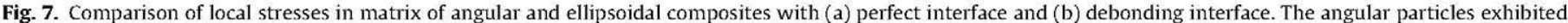
higher stress due to more load transfer, and relaxation after debonding. The macroscopic applied strain is $1.9 \%$.

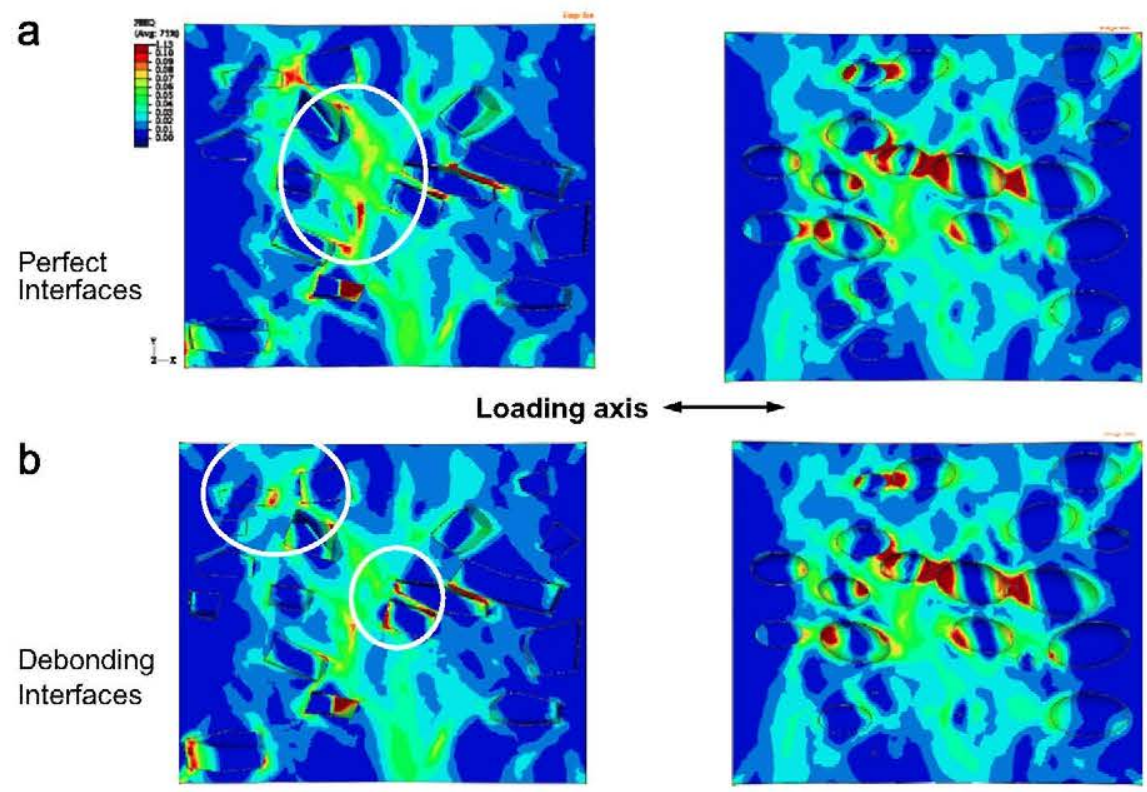

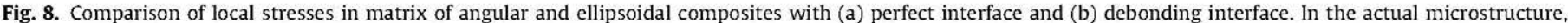
plasticity shifts from the matrix-rich regions (for perfect interface) to the particle/matrix interface (after debonding). The macroscopic applied strain is $1.9 \%$. 
on the face $x_{1}=0$ were pinned at zero displacement in all the three directions.

The effect of interface strength, using cohesive zone elements, for both angular and ellipsoid particles is shown in Fig. 5. The following interface strengths were employed, $300 \mathrm{MPa}, 500 \mathrm{MPa}, 3000 \mathrm{MPa}$, and a perfect interface (infinite strength). In the actual microstructure, the decrease in composite strength is quite significant. In the ellipsoid case only a small change is observed, which occurs at the lowest interface strength of $300 \mathrm{MPa}$. The mechanisms by which this dramatic change takes place can be described as follows. We can first examine the degree of load transfer for both types of particles. The angular particles provide more flat surfaces for load transfer, and thus, these particles are more conducive to carrying load transferred from the matrix. Sharp stresses at particle corners are also observed, which dramatically increase the triaxial stress in the matrix. Note that with debonding the stress of the particles decreases significantly. This is shown in two ways. Fig. 6 shows a 3D view of just the $\mathrm{SiC}$ particles, in both perfectly bonded and debonded cases. Fig. 7 shows the particles and matrix, but in a 2D section of the composite, again, for perfectly bonded and debonded cases. It is interesting to note that in the angular particles, the surfaces that are normal to the loading axis carry load. Thus, when debonding takes place, these surfaces are relieved of the stress and relaxation takes place. Such a transition during debonding is not observed in the ellipsoidal particles, because of the smooth overall shape and absences of flat surfaces. This explains the lack of sensitivity to debonding observed in the ellipsoidal particle reinforced composites.

The location of highest strain localization and plasticity also is different for perfect bonding and debonded cases in the composite with angular particles. In the perfectly bonded case plasticity is highest in the matrix-rich regions of the composite (Fig. 8). After debonding, the strain intensification is right at the particle/ matrix interface. The differences in ellipsoidal particle composite are not as pronounced.

\section{Summary}

We have studied the effect of particle/matrix interface debonding in SiC particle reinforced Al alloy matrix composites with (a) actual microstructure consisting of angular SiC particles and (b) idealized ellipsoidal SiC particles. Tensile deformation in $\mathrm{SiC}$ particle reinforced $\mathrm{Al}$ matrix composites was modeled using actual microstructures reconstructed from serial sectioning approach. Interfacial debonding was modeled using user-defined cohesive zone elements.
Modeling with the actual microstructure (versus idealized ellipsoids) has a significant influence on: (a) localized stresses and strains in particle and matrix, and (b) far-field strain at which localized debonding takes place. The angular particles exhibited higher degree of load transfer and are more sensitive to interfacial debonding. Larger decreases in stress are observed in the angular particles, because of the flat surfaces, normal to the loading axis, which bear load. Furthermore, simplification of particle morphology may lead to erroneous results.

\section{References}

[1] J. Llorca, A. Needleman, S. Suresh, Scr. Metall. Mater. 24 (1990) 1203-1208.

[2] J.R. Brockenbrough, S. Suresh, H.A. Wienecke, Acta Metall. Mater. 39 (1991) 735-752.

[3] Y.L. Shen, M. Finot, A. Needleman, S. Suresh, Acta Metall. Mater. 42 (1994) 77-97.

[4] X. Deng, N. Chawla, J. Mater. Sci. 41 (2006) 5731-5734.

[5] J. LLorca, C. González, J. Mech. Phys. Solids 46 (1998) 1-28.

[6] J. LLorca, P. Poza, Acta Metall. Mater. 43 (1995) 3959-3969.

[7] D. Steglich, T. Siegmund, W. Brocks, Comput. Mater. Sci. 16 (1999) 404-413.

[8] J. Llorca, A. Needleman, S. Suresh, Acta Metall. Mater. 39 (1991) 2317-2335.

[9] S.R. Nutt, A. Needleman, Scr. Metall. Mater. 21 (1987) 705-710.

[10] M. Li, S. Ghosh, T.N. Rouns, H. Weiland, O. Richmond, W. Hunt, Mater. Charact. 41 (1998) 81-95.

[11] M. Li, S. Ghosh, O. Richmond, Acta Mater. 47 (1999) 3515-3532.

[12] S. Ghosh, S. Moorty, Comput. Mech. 34 (2004) 510.

[13] J. Boselli, P.D. Pitcher, P.J. Gregson, I. Sinclair, Mater. Sci. Eng. A 300 (2001) 113-124.

[14] J. Segurado, C. Gonzalez, J. Llorca, Acta Mater. 51 (2003) 2355-2369.

[15] J. Segurado, J. Llorca, Acta Mater. 53 (2005) 4931-4942

[16] J. Llorca, J. Segurado, Mater. Sci. Eng. A 365 (2004) 267-274.

[17] J. Segurado, J. LLorca, Mech. Mater. 38 (2006) 873-883.

[18] N. Chawla, B.V. Patel, M. Koopman, K.K. Chawla, R. Saha, B.R. Patterson, E.R. Fuller, S.A. Langer, Mater. Charact. 49 (2003) 395-407.

[19] V.V. Ganesh, N. Chawla, Mater. Sci. Eng. A 391 (2005) 342-353.

[20] N. Chawla, V.V. Ganesh, B. Wunsch, Scr. Mater. 51 (2004) 161-165.

[21] Z. Shan, M. Gokhale, Acta Mater. 49 (2001) 2001-2015.

[22] L. Babout, E. Maire, R. Fougeres, Acta Mater. 52 (2004) 2475-2487.

[23] A. Borbely, F.F. Csikor, S. Zabler, P. Cloetens, H. Biermann, Mater. Sci. Eng. A 367 (2004) 40-50.

[24] J.J. Williams, Z. Flom, A.A. Amell, N. Chawla, X. Xiao, F. De Carlo, Acta Mater 58 (2010) 6194-6205.

[25] F.A. Silva, J.J. Williams, B.R. Mueller, M.P. Hentschel, P.D. Portella, N. Chawla, Metall. Mater. Trans. A 41A (2010) 2121-2128.

[26] P. Kenesei, H. Biermann, A. Borbely, Scr. Mater. 53 (2005) 787-791.

[27] L.L. Mishnaevsky Jr., Mater. Sci. Eng. A 407 (2005) 11-23.

[28] H.R. Lusti, P. Hine, A.A. Gusev, Compos. Sci. Technol. 62 (2002) 1927-1934.

[29] A.A. Gusev, H.R. Lusti, P.J. Hine, Adv. Eng. Mater. 4 (2002) 927-931.

[30] N. Lippmann, Th. Steinkopff, S. Schmauder, P. Gumbsch, Comput. Mater. Sci. 9 (1997) 28-35

[31] A. Mortensen, J. Llorca, Annu. Rev. Mater. Res. 40 (2010) 243-270.

[32] D.J. Lloyd, in: P.K. Mallick (Ed.), Composite Engineering Handbook, Marcel Dekker, New York, NY, 1997, pp. 631-670.

[33] J. Segurado, J. LLorca, Int. J. Solids Struct. 41 (2004) 2977-2993. 